\title{
An Initiative to Build Research Capacity within a Physiotherapy Department: Hits and Misses Over a 20-year Period
}

\author{
Kathy Stiller \\ Central Adelaide Local Health Network, kathy.stiller@sa.gov.au \\ Naomi Haensel \\ Central Adelaide Local Health Network, naomi.haensel@sa.gov.au
}

Follow this and additional works at: https://nsuworks.nova.edu/ijahsp

Part of the Physiotherapy Commons

\section{Recommended Citation}

Stiller K, Haensel N. An Initiative to Build Research Capacity within a Physiotherapy Department: Hits and Misses Over a 20-year Period. The Internet Journal of Allied Health Sciences and Practice. 2016 Jan 01;14(4), Article 1.

This Manuscript is brought to you for free and open access by the College of Health Care Sciences at NSUWorks. It has been accepted for inclusion in Internet Journal of Allied Health Sciences and Practice by an authorized editor of NSUWorks. For more information, please contact nsuworks@nova.edu. 


\title{
An Initiative to Build Research Capacity within a Physiotherapy Department: Hits and Misses Over a 20-year Period
}

\begin{abstract}
Purpose: Building research capacity within a busy clinical allied health department is important but can be limited by time, resources and expertise. In 1995, a role was created in our Physiotherapy Department whereby an individual with an interest and expertise in clinical research was identified and a proportion of their workload (12 hours/week) designated to fulfil a research co-ordination role. The aim of this role was to foster research and build research capacity within the Physiotherapy Department. This paper reports the achievements associated with this initiative to highlight its successes, difficulties and failures. Method: A retrospective descriptive review of research and other research-related activities undertaken over a 20-year period within the Physiotherapy Department of an adult, tertiary, public hospital was carried out, with particular attention to successes, difficulties and failures. Results: Since the introduction of a designated research co-ordinator in 1995, there have been 54 major publications in peer-reviewed journals and 91 presentations at a national/international conferences. Successes have included the high number of publications/presentations, the broad range of clinical areas where research has been undertaken, the clinical relevance of the research, the large number of staff involved, collaboration with other hospital departments/universities and staff involvement in post-graduate degrees. Difficulties have included slow recruitment rates to clinical trials, resource issues and lack of career structure for those interested in pursuing research as a career option within the healthcare sector. Failures have been infrequent but have included the need to abandon three studies due to recruitment or personnel issues, and a single instance of the inadvertent use of incorrect data. Conclusions: Identifying a clinician with an interest and expertise in clinical research and quarantining time to enable them to fulfil a broader research co-ordinating role was successful at fostering research and building research capacity within a clinical allied health department.
\end{abstract}

\section{Author Bio(s)}

Kathy Stiller, B App Sc (Physio), PhD, is the Allied Health Research Co-ordinator at Central Adelaide Local Health Network.

Naomi Haensel, Dip Tech (Physio), Grad Dip (HIth Admin), is Senior Manager, Physiotherapy, Central Adelaide Local Health Network. 


\title{
IJAHSP \\ The Internet Joưnal of Allied Health Sciences and Practice
}

Dedicated to allied health professional practice and education

Vol. 14 No. 4 ISSN 1540-580X

\section{An Initiative to Build Research Capacity within a Physiotherapy Department: Hits and Misses Over a 20-year Period}

\author{
Kathy Stiller, B App Sc (Physio), PhD \\ Naomi Haensel, Dip Tech (Physio), Grad Dip (Hlth Admin) \\ Central Adelaide Local Health Network
}

Australia

\begin{abstract}
Purpose: Building research capacity within a busy clinical allied health department is important but can be limited by time, resources, and expertise. In 1995, a role was created in our Physiotherapy Department whereby an individual with an interest and expertise in clinical research was identified and a proportion of their workload (12 hours/week) designated to fulfil a research coordination role. The aim of this role was to foster research and build research capacity within the Physiotherapy Department. This paper reports the achievements associated with this initiative to highlight its successes, difficulties, and failures. Method: A retrospective descriptive review of research and other research-related activities undertaken over a 20-year period within the Physiotherapy Department of an adult, tertiary, public hospital was carried out, with particular attention to successes, difficulties, and failures. Results: Since the introduction of a designated research coordinator in 1995, there have been 54 major publications in peer-reviewed journals and 91 presentations at national/international conferences. Successes have included the high number of publications/presentations, the broad range of clinical areas where research has been undertaken, the clinical relevance of the research, the large number of staff involved, collaboration with other hospital departments/universities, and staff involvement in post-graduate degrees. Difficulties have included slow recruitment rates to clinical trials, resource issues, and lack of career structure for those interested in pursuing research as a career option within the healthcare sector. Failures have been infrequent but have included the need to abandon three studies because of recruitment or personnel issues, and a single instance of the inadvertent use of incorrect data. Conclusions: Identifying a clinician with an interest and expertise in clinical research and quarantining time to enable them to fulfil a broader research coordinating role was successful at fostering research and building research capacity within a clinical allied health department.
\end{abstract}

\section{INTRODUCTION}

Evidence-based practice informs clinical decision-making and policy in healthcare, with the aim of optimizing patient outcomes whilst promoting the effective and efficient use of limited healthcare resources. To enable evidence-based practice, it is vital that clinical research that is patient-centered and relevant to clinical practice is undertaken. In contrast to medical research, which has been established and well recognized for decades, the advent of allied health research has been relatively recent and often driven under the auspices of universities rather than being generated from within clinical allied health departments. ${ }^{1}$ The involvement of allied health clinicians in research is very important as it encourages critical thinking, develops and promotes a research culture, and allows clinicians the ability to provide input into the design of research such that it addresses pertinent clinical questions. ${ }^{1}$

Clinicians' motivations for undertaking clinical research include development of research skills, increased job satisfaction, and the ability to investigate clinical problems that are relevant to practice. ${ }^{2,3}$ Barriers to undertaking research within clinical departments include workplace issues such as a lack of time/funding, higher priority of other work roles, lack of research skills and/or access to people with relevant research expertise, inadequate career structure for researchers, and a poor research culture within clinical departments. ${ }^{2-7}$

Various methods for building research capacity have been described and include:

- developing a workplace culture that recognizes the importance of research as an integral part of clinical practice

(c) The Internet Journal of Allied Health Sciences and Practice, 2016 
- clear leadership that supports and promotes clinical research

- increased workplace and career flexibility to allow clinicians time to participate in research

- funding through grants, scholarships, and bursaries

- collaboration with universities and other external bodies to co-ordinate, provide, and support research training

- identifying individuals with an interest in research and empowering them to build their own research expertise and that of their colleagues through devoted resources

- improved communication about research activities and findings across the organization. 1,3,4-11

Successful building of research capacity in individuals and organizations leads to a workplace culture where research is actively discussed and seen as an integral part of clinicians' roles, higher levels of research skills, and a greater ability of clinicians to undertake relevant research on a consistent basis. ${ }^{10,11}$

Within our own health unit, our anecdotal experience was that whilst clinical research had been actively encouraged within our Physiotherapy Department for a number of years, success was limited in terms of the number and scope of projects undertaken and the number of resultant publications and presentations. Therefore, in 1995, a role was created in our Physiotherapy Department whereby an individual with an interest and expertise in clinical research was identified and a proportion of their workload designated as fulfilling a research coordination role across our Physiotherapy Department, with the intention of fostering clinical research and building research capacity within our Department. The aim of this paper is to review the achievements associated with this initiative to highlight its successes, difficulties, and failures.

\section{METHODS}

\section{Design and Timeline}

A retrospective descriptive review was undertaken of research activity over a 20-year period (1995 to 2014 inclusive).

\section{Setting and Timeline}

This study involved reviewing research and research-related activities undertaken within the Physiotherapy Department, Royal Adelaide Hospital, and Hampstead Rehabilitation Centre since the commencement of this initiative to build research capacity (1995) up to the point in time where the position was expanded from being physiotherapy-only to a broader allied health role (2015). The Royal Adelaide Hospital is an adult, 650-bed, tertiary-care, urban, public hospital in Australia. Hampstead Rehabilitation Centre is a 128-bed campus of the Royal Adelaide Hospital that provides rehabilitation to patients following stroke, brain injury, spinal cord injury, other neurological or medical disorders, orthopedic conditions, amputation or burn injury. The Physiotherapy Department provides comprehensive in- and out-patient services across both campuses.

\section{Background to the Creation of the Research Coordinator Role}

Prior to 1995, Royal Adelaide Hospital Physiotherapy Department staff were encouraged to be involved in clinical research, including by the introduction of project rosters whereby staff wishing to undertake research in a particular clinical area were able to remain in this area beyond the usual rotational time. This approach achieved modest success, with physiotherapy staff generating 14 publications in peer-reviewed medical/physiotherapy journals prior to 1995. However, the Director of Physiotherapy $(\mathrm{NH})$ believed additional action was required to further build research capacity within the Department and took advantage of an opportunity that was rare for the time as follows: One staff member (KS) had demonstrated a particular interest in clinical research, having been involved in 11 of the 14 publications generated between 1987-1994 and successfully completing a Doctor of Philosophy $(\mathrm{PhD})$ degree, based on two clinical studies, whilst continuing to work full time. This staff member's PhD candidature had been supported at a departmental level, including recognition that involvement in clinical research should be seen as an integral part of a clinician's role and allowing a proportion of her workload to be devoted to the clinical research comprising her $\mathrm{PhD}$ candidature. Following the successful completion of this staff member's PhD degree, the Director $(\mathrm{NH})$ proposed that this staff member should continue to undertake clinical research within the Royal Adelaide Hospital Physiotherapy Department, and importantly, expand this research role from her own area of expertise (cardiorespiratory) to other areas of adult physiotherapy clinical practice. This initiative was supported by Royal Adelaide Hospital executive staff who acknowledged it as "needed if allied health departments in general, and physiotherapy in particular, are to raise their profile with medical and nursing colleagues" (personal communication, 1995). Thus, the role of Research Co-ordinator, Physiotherapy, was officially created and recognized in 1995, with 12 hours/week quarantined for fulfilling this role, the aim of which was to foster clinical research and build research capacity within the Royal Adelaide Hospital Physiotherapy Department.

\section{Outcomes}

For the purposes of this report, the effectiveness of this initiative to build research capacity was primarily measured by the number of major publications in peer-reviewed journals and presentations at national/international conferences. Minor publications (e.g.,

(c) The Internet Journal of Allied Health Sciences and Practice, 2016 
publications in non-peer-reviewed journals, letters to the editor, and book reviews) were excluded, and only research that was undertaken by physiotherapy staff as part of their Royal Adelaide Hospital role was included. Additional outcomes included the conversion rate of projects that were commenced to publication/presentation, the range of clinical areas where research was undertaken, the clinical relevance of the research, the number of staff involved and their level of experience, the extent of collaboration with other departments/universities, the number of staff who undertook research as part/all of post-graduate research degrees, and the number of successful grant applications.

These data were retrieved from the yearly research reports and an ongoing research register that was commenced by the Research Co-ordinator (KS) at the inception of the position in 1995. Data were reviewed by the two investigators, with any disagreement resolved through consensus. Additionally, as the two investigators were personally involved with this initiative throughout the 20year period, anecdotal recollections have, when appropriate, been included.

\section{Data Analysis}

Data were collated and analyzed descriptively, with successes, difficulties, and failures highlighted.

\section{RESULTS}

\section{Successes}

The research undertaken over the 20-year period as a result of this initiative generated a total of 54 major publications in peerreviewed journals and 91 presentations at national/international conferences, resulting in a median of two publications and five presentations per year. Figure 1 shows these publications/presentations on a yearly basis since inception of this initiative. Whilst journal impact factors were not specifically recorded at the time, the journals involved comprised mainly physiotherapy journals (e.g., Journal of Physiotherapy, Physiotherapy Research International, Physiotherapy Theory and Practice) and some medical journals (e.g., Archives of Physical Medicine and Rehabilitation, Chest, Spinal Cord). The conferences where research findings were presented were most often national/international physiotherapy conferences (e.g., Australian Physiotherapy Association Conference, World Confederation for Physical Therapy) with additional presentations at international medical conferences (e.g., Australian and New Zealand Cystic Fibrosis Conference, Australian and New Zealand Burn Association Annual Scientific Meeting, International Symposium on Intensive Care and Emergency Medicine). The most common types of research undertaken during the 20-year period of this initiative were clinical studies $(n=42)$, with literature reviews the next most frequent $(n=5)$.

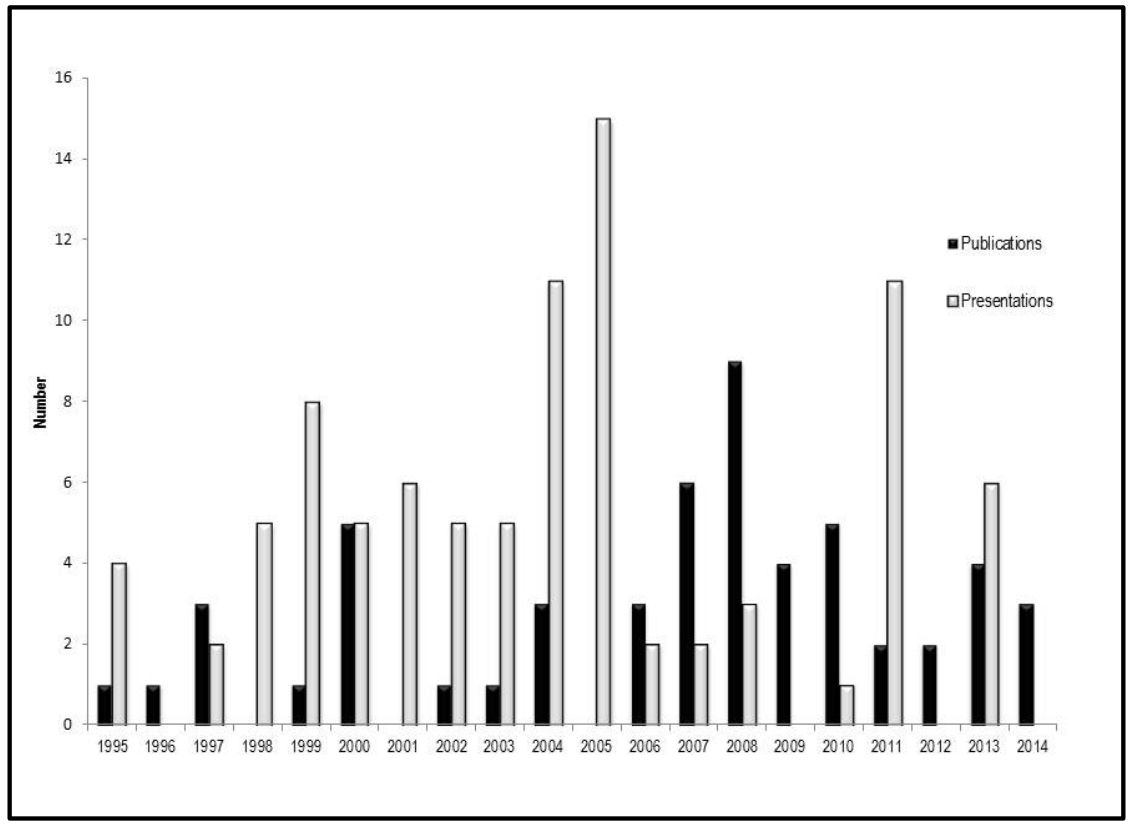

Figure 1: National/international publications and presentations on a yearly basis

In terms of the conversion rate for research to publication/presentation, a 100\% rate was achieved for studies where data collection was completed over the 20-year period. In other words, all studies where data collection was completed achieved publication and/or presentation. 
The range of clinical areas in which research was undertaken was broad (see Figure 2), involving most areas of adult physiotherapy practice, with intensive care, stroke rehabilitation, post-operative management, cystic fibrosis, and spinal cord injury rehabilitation occurring most frequently.

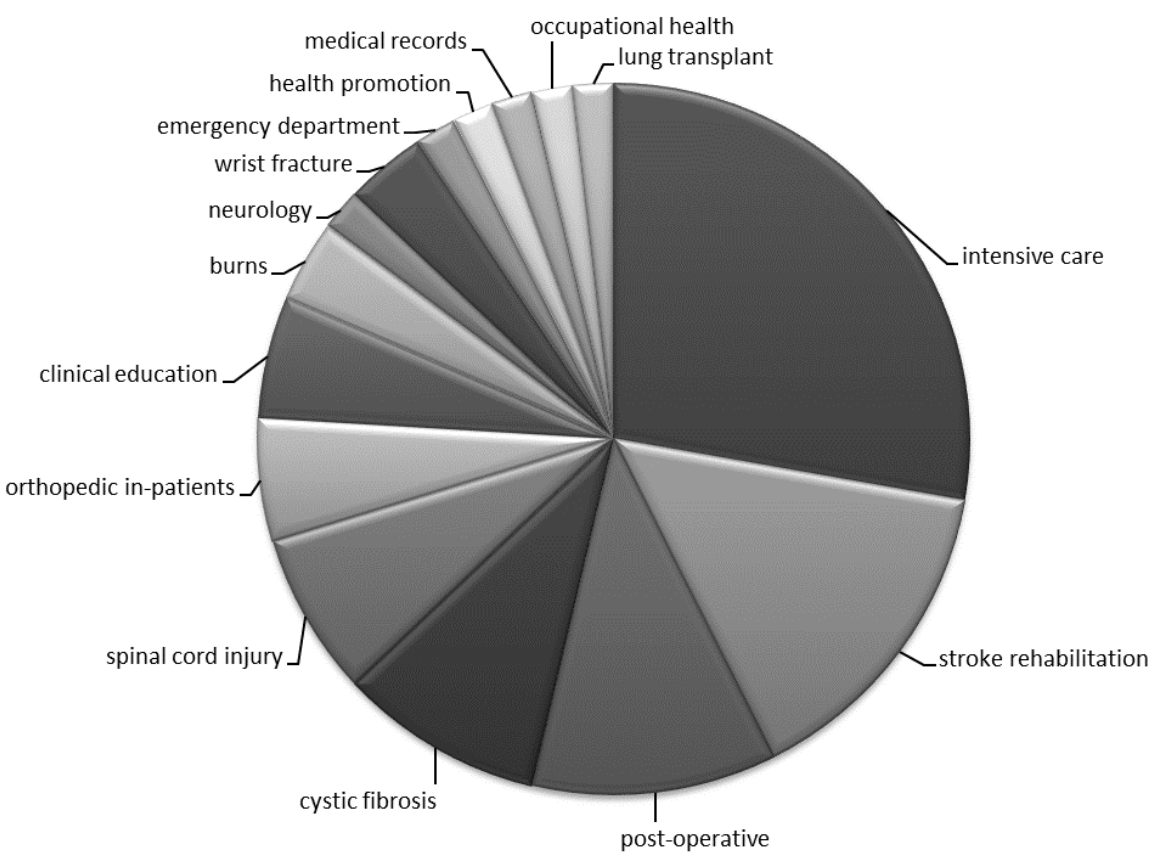

Figure 2: Clinical areas in which physiotherapy research was undertaken

Of the research undertaken, virtually all projects were patient-centered, driven by Royal Adelaide Hospital Physiotherapy Department staff members, clinically relevant and influenced clinical practice within our health unit, and in some instances, internationally. Examples of this were studies investigating whether bed exercises are necessary after hip arthroplasty, safety aspects of mobilizing critically ill patients, the effectiveness of circuit class therapy during stroke rehabilitation, physical outcomes of patients with burn injuries, and the effectiveness of physiotherapy interventions for patients with distal radius fracture. ${ }^{12-17}$

Thirty-four Royal Adelaide Hospital Physiotherapy Department staff were involved in the research undertaken over the term of this initiative, with their level of experience ranging from newly graduated physiotherapists to senior physiotherapists. Some of these staff members $(n=14)$ had repeated involvement in research projects over this time period, demonstrating the success of this initiative at fostering an ongoing interest and commitment to clinical research. Anecdotally, our experience suggested that it was more effective and efficient to involve and increase the research knowledge/expertise only for those staff who expressed an interest in undertaking clinical research, rather than attempting to involve/educate all clinicians (e.g., through a series of presentations about research methodology to all staff). The level of support required by staff during the research process varied considerably and thus they were provided with as much or as little support as required on an individual basis. For novice researchers, our experience suggested that it was best to involve them in studies with relatively simple research designs, rather than launching into a randomized controlled trial as an initial step.

Some of the research that was undertaken as part of this initiative involved collaboration with other departments within the Royal Adelaide Hospital $(n=5)$, with the departments involved including the Burns Unit, Intensive Care Unit, Stroke Rehabilitation Unit, and Radiology Department. Collaboration with external institutions also occurred quite frequently $(n=27)$, including, for example, with the University of South Australia, Curtin University, and University of Melbourne.

Of the research undertaken or commenced within this time period, nine Physiotherapy Department staff members undertook the research as part (or all) of a research degree, comprising five Honours, two Masters, and two PhD degrees. The Research Coordinator (KS) was a co-supervisor for all of these staff members during their research degree candidature. Additionally, the Research Coordinator (KS) was a co-supervisor for two physiotherapists who were external to the Royal Adelaide Hospital: one 
physiotherapist undertook a Masters, then PhD, through Curtin University, and the other physiotherapist undertook a PhD through the University of Melbourne.

Nine grant applications were successful over the 20-year initiative, totalling approximately $\$ 70,000$. Two grants were from the Physiotherapy Research Foundation and the other five were sourced from internal Royal Adelaide Hospital grants. Other successes achieved as a result of this research building initiative included the recognition by hospital and research executives within the Royal Adelaide Hospital of the need for specific allied health grants to fund allied health research. As a result, specific funds were identified from within the suite of research grants supported by the organization - these continue to provide support for allied health research.

Another major success associated with this initiative was the national and international recognition of the research undertaken within the Physiotherapy Department, Royal Adelaide Hospital. This was evident in, for example, the Research Coordinator (KS) being invited to present at national/international physiotherapy/medical conferences as a keynote speaker on 17 occasions, her invited participation in an international taskforce regarding physiotherapy for adult patients with critical illness, and ongoing requests and involvement in reviewing national/international grant applications and journal submissions. ${ }^{18}$ Whilst impossible to quantify, we believe that another successful outcome of this research building initiative has been that staff working within our Physiotherapy Department have an increased awareness of clinical research and evidence-based practice, thus providing a clear rationale for our clinical services in a resource-constrained environment. Finally, the sustained success of this initiative over a 20 -year period has led to a somewhat bittersweet development, with the position now being widened from physiotherapy-only to a broader role across allied health for the Central Adelaide Local Health Network.

\section{Difficulties}

Initially, when this research capacity building initiative was commenced in 1995, there was some discontent amongst peers within the Royal Adelaide Hospital Physiotherapy Department who viewed the conversion of clinical time to clinical research as a luxury. This concern lessened over time as the successes associated with the initiative became evident.

The most frequent difficulty encountered over the 20-year period of this initiative was slow recruitment rates to clinical trials, with all clinical studies experiencing this difficulty. This led to a re-focusing of clinical research to preferentially target high-volume clinical areas. Resource issues were also frequently encountered, with time constraints adversely affecting the research process. Indeed, even when grant money enabled back-filling so that clinicians could have dedicated research time, it was often difficult back-filling to these part-time and temporary positions. Another difficulty encountered during this initiative was the occasional lack of engagement from non-physiotherapy staff. For example, a slow response rate by non-physiotherapy staff to requests to review research proposals or manuscripts led to delays in the research process. Another area where our research capacity building initiative experienced difficulty was in trying to disseminate the successes of the initiative to non-physiotherapist professional groups, with no clear avenues available to do this outside of publications/presentations.

The lack of career opportunities for staff who had been involved in the research projects and wished to further develop their expertise became evident over time. In some instances $(n=5)$, this led to these staff relinquishing their positions and moving to university positions. This in itself led to a further difficulty, in that the loss of key staff with specific research interest and expertise resulted in an over-reliance on the Research Coordinator (KS) to assist with most, if not all, phases of the research process in order for it to be successfully completed. Thus, whilst an aim of the initiative was to build the ability of staff members to engage in and conduct all aspects of the research process with increasing independence, we believe this was only partially successful.

\section{Failures}

Failures associated with this research building initiative were infrequent and included the need to abandon three clinical studies: on two occasions because of inordinately slow recruitment rates, and on the other occasion due to personnel issues. Another failure involved a single occasion when disease severity data, obtained from an external source, were found to be inaccurate during manuscript revision for a journal, requiring complete re-analysis and re-writing of the paper as it substantially altered the findings. Finally, despite some informal overtures regarding a joint appointment with a university, these weren't pursued and so no formal partnership with the tertiary education sector was formed.

\section{DISCUSSION}

This initiative, whereby an individual with an interest and expertise in clinical research was identified and a proportion of their workload quarantined as fulfilling a research co-ordination role, was extremely successful at fostering research and building research capacity within a busy physiotherapy department, as measured by outcomes including the number of publications/presentations, range of research, clinical relevance and number of staff involved.

(C) The Internet Journal of Allied Health Sciences and Practice, 2016 
Because of a lack of data regarding initiatives to build research capacity in a clinical allied health department, it is not possible to compare the specific outcomes associated with this initiative to similar initiatives or alternative methods of building research capacity. Pager et al noted that supporting already motivated individuals and teams to conduct research is likely to produce better outcomes for research capacity building investment than a more generalized approach. ${ }^{2}$ Our findings concur with this in that we believe an important reason for the success of our initiative was that rather than attempting to engage all staff in research, we instead sought the involvement of those with an interest in clinical research. Whilst our initiative was structured around providing increased resources/expertise for clinical research, it achieved other successes that have been shown to be important in building research capacity, including enhancing organizational approach and support from senior managers, improving research culture whereby research is seen as part of a clinician's role, building partnerships and collaboration, and providing new opportunities for staff involvement in clinical research. ${ }^{8}$ The cost associated with this research co-ordination role over the 20-year period was approximately $\$ A 450,000$ or $\$ A 22,500$ per year but was achieved by a re-direction of existing resources rather than new funding. We believe this was money well spent in terms of building current and future physiotherapy research capacity as measured by the number of physiotherapists who gained research experience, growing research grant success and the recognition within the Royal Adelaide Hospital of the value of allied health research leading to the establishment of specific allied health grants.

Some of the lessons that we learned as a result of this initiative merit further discussion. We believe the success of this initiative to build research capacity reflected the ability of the person who was appointed as Research Coordinator (KS) rather than the position per se. Clearly, for such an initiative to be successful, it is paramount that the right person be appointed. We believe that key personal attributes for a position such as this include enthusiasm, clear-sightedness, adaptability, and dogged perseverance, along with demonstrated expertise in all phases of clinical research. We also believe that the clear link between the research that was undertaken and clinical practice was an important factor contributing to its success. Involving only those staff who indicated an interest in clinical research and preferentially focusing on clinical areas with relatively high-volumes of patients were also key to the success of the initiative. Another important lesson that we learned, fortunately early on, was to keep a comprehensive list and register of all research-related activities (e.g., titles of all projects commenced and the names of staff involved; lists of ethics applications, clinical trials registrations, publications, presentations, grant applications) as this allowed us to assess the effectiveness of our initiative to build research capacity.

\section{Limitations}

The results of this retrospective review are only directly applicable to our Physiotherapy Department, and clearly, any interpretations by the authors have been influenced by our personal involvement in the initiative. Nevertheless, we believe the achievements associated with this initiative will be of interest to other allied health practitioners and allied health departments seeking to build research capacity. Whilst our initiative was structured around quarantining time to enable a person to fulfill a research coordination role, we acknowledge that other methods of building research capacity may be equally effective. We also acknowledge that it would have been preferable if we had recorded, at the time, the impact factor of journals where publications occurred. However, we believe that to build research capacity in a clinical department, novice researchers appreciate publication and the impact factor of the journal is not the primary consideration.

\section{Conclusions}

Identifying a clinician with an interest and expertise in clinical research and quarantining time to enable them to fulfil a broader research coordinating role was successful at fostering research and building research capacity within an allied health clinical department. This mentoring role enabled numerous physiotherapy staff who previously had limited research expertise to successfully undertake and complete research.

\section{REFERENCES}

1. Pickstone C, Nancarrow S, Cooke J, Vernon W, Mountain G, Boyce R, Campbell J. Building research capacity in the allied health professions. Evidence and Policy. 2008:4(1):53-68.

2. Pager S, Holden L, Golenko X. Motivators, enablers, and barriers to building allied health research capacity. J Multidiscip Healthc. 2012;5:53-9. [PMID 22396626]

3. Ried K, Farmer EA, Weston KM. Bursaries, writing grants and fellowships: a strategy to develop research capacity in primary health care. BMC Fam Pract. 2007;8:19. [PMID 17408497]

(C) The Internet Journal of Allied Health Sciences and Practice, 2016 
4. Frontera WR, Fuhrer MJ, Jette AM, Chan L, Cooper RA, Duncan PW, Kemp JD, Ottenbacher KJ, Peckham PH, Roth EJ, Tate DG. Rehabilitation medicine summit: building research capacity. Executive summary. Arch Phys Med Rehabil. 2006;87(1):148-52. [PMID 16401455]

5. Grange A, Herne S, Casey A, Wordsworth L. Building research capacity. Nurs Manag (Harrow). 2005;12(7):32-7. [PMID 16295618]

6. Janssen J, Hale L, Mirfin-Veitch B, Harland T. Building the research capacity of clinical physical therapists using a participatory action research approach. Phys Ther. 2013;93(7):923-34. [PMID 23559527]

7. Segrott J, Mclvor M, Green B. Challenges and strategies in developing nursing research capacity: a review of the literature. Int J Nurs Stud. 2006;43(5):637-51. [PMID 16157338]

8. Golenko X, Pager S, Holden L. A thematic analysis of the role of the organisation in building allied health research capacity: a senior managers' perspective. BMC Health Serv Res. 2012;12:276. [PMID 22920443]

9. Hulcombe J, Sturgess J, Souvlis T, Fitzgerald C. An approach to building research capacity for health practitioners in a public health environment: an organisational perspective. Aust Health Rev. 2014;38(3):252-8. [PMID 24785693]

10. Kumar S, Ducat W. Building research capacity in regional, rural and remote allied health services: lessons from evidence and experience. Internet J Allied Health Sciences Practice. 2014;12(3).

11. Murphy K, Stockton D, Kolbe A, Hulme-Chambers A, Smythe G. Building research capacity in a regional Australian health service: a management strategy analysis. Asia Pac J Health Manage. 2015;10(1).

12. English C, Hillier S, Stiller K, Warden-Flood A. Circuit class therapy versus individual physiotherapy sessions during inpatient stroke rehabilitation: a controlled trial. Arch Phys Med Rehabil. 2007;88(8):955-63. [PMID 17678655]

13. Jarrett M, McMahon M, Stiller K. Physical outcomes of patients with burn injuries - a 12 month follow-up. J Burn Care Res. 2008;29(6):975-84. [PMID 18849834]

14. Jesudason C, Stiller K. Are bed exercises necessary following hip arthroplasty? Aust J Physiother. 2002;48(2):73-81. [PMID 12047205]

15. Kay S, Haensel N, Stiller K. The effect of passive mobilisation following fractures involving the distal radius: a randomised study. Aust J Physiother. 2000;46(2):93-101. [PMID 11676793]

16. Kay S, McMahon M, Stiller K. An advice and exercise program has some benefits over natural recovery after distal radius fracture: a randomised trial. Aust J Physiother. 2008;54(4):253-9. [PMID 19025505]

17. Stiller K, Phillips A. Safety aspects of mobilising acutely ill in-patients. Physiother Theory Practice. 2003;19:239-57.

18. Gosselink R, Bott J, Johnson M, Dean E, Nava S, Norrenberg M, Schonhofer B, Stiller K, van der Leur H, Vincent JL. Physiotherapy for adult patients with critical illness: recommendations of the European Respiratory Society and European Society of Intensive Care Medicine Task Force on physiotherapy for critically ill patients. Intensive Care Med. 2008;34(7):1188-99. [PMID 18283429] 